\title{
Electrochemical Detection of Curcumin in Food with a Carbon Nanotube-Carboxymethylcellulose Electrode
}

\author{
Ryotaro Wada,* Shota TaKahashi, ${ }^{*}$ Hitoshi Muguruma, ${ }^{* \dagger}$ and Naomi OsaKabe** \\ *Graduate School of Engineering and Science, Shibaura Institute of Technology, 3-7-5 Toyosu, Koto, \\ Tokyo 135-8548, Japan \\ **Department of Bioscience and Engineering, College of Systems Engineering and Science, \\ Shibaura Institute of Technology, 307 Fukasaku, Minuma, Saitama 337-8570, Japan
}

\begin{abstract}
Herein, an electrochemical method is presented for the detection of curcumin in food using a carbon nanotube (CNT)carboxymethylcellulose (CMC) electrode. The CNT-CMC electrode exhibited ideal characteristics for curcumin detection, namely, a high response current and adequate peak separation toward curcumin oxidation. Cyclic voltammetry revealed two oxidation peaks. In the first scan, only the irreversible peak (Peak I) was observed at a higher potential. In the second scan, the reversible redox peak pairs (Peaks II and $\mathrm{II}^{\prime}$ ) appeared at lower potentials, and the potential of Peak I was decreased. Peak I corresponded to oxidation of the hydroxyl groups of the benzene ring to the catechol group via a phenoxy radical, while Peaks II and II' indicated the redox loop system of the generated catechol group. The current at Peak II was used to quantify the concentration of curcumin in the linear range of $1-48 \mu \mathrm{M}$ and detection limit of $0.084 \mu \mathrm{M}$. The concentrations of curcumin determined by the CNT-CMC electrode in real food samples were consistent with those determined by high-performance liquid chromatography.
\end{abstract}

Keywords Carbon nanotube, cyclic voltammetry, curcumin, redox reaction

(Received January 22, 2020; Accepted April 24, 2020; Advance Publication Released Online by J-STAGE May 1, 2020)

\section{Introduction}

Curcumin, chemically called 1,7-bis(4-hydroxy-3-methoxyphenyl)1,6-heptadieno-3,5-dinone, is a natural polyphenol and a yellow pigment obtained from the rhizome of turmeric (Curcuma longa Linn.). Its chemical structure is shown in Fig. 1. It is often used in various cuisines as a spicing, flavoring, or coloring agent. The intake of curcumin has numerous health advantages, including anti-tumour, anti-arthritic, anti-inflammatory, anticancer, and anti-HIV activities, and the reduction of obesity., These effects are attributed to the antioxidant capacity or scavenging of reactive oxygen species by curcumin. Curcumin can chelate various metal ions. Several methods have been reported for the determination of curcumin, such as spectroscopy, ${ }^{3}$ resonance light scattering, ${ }^{4}$ and high-performance liquid chromatography (HPLC). ${ }^{5}$ However, these methods require bulky apparatus and are sophisticated, time-consuming, and expensive. Based on these drawbacks, an electrochemical method for the detection and quantification of curcumin is promising. However, there are only a few reports concerning electrochemical analysis of curcumin, and the electrochemistry of curcumin is still unclear. ${ }^{6-12}$

In this study, carbon nanotube (CNT)-based electrodes were chosen for the electrochemical analysis of curcumin because of their excellent electrochemical behavior. There are many reports concerning polyphenol analysis with CNT-base electrodes. ${ }^{13-16}$

† To whom correspondence should be addressed.

E-mail: muguruma@shibaura-it.ac.jp
In contrast, there has been only one report on curcumin sensing with CNT-based electrodes, ${ }^{9}$ likely because of the hydrophobic nature of the CNT electrode and the fact that as-synthesised CNT is an agglomeration of molecules. In a previous study, we reported on an electrode with CNT-carboxymethyl cellulose (CMC) dispersion. ${ }^{17,18}$ Herein, we have attempted to expand the applicability of the CNT-CMC electrode. In the CNT-CMC dispersion, the negatively charged CMC molecules unbundle the CNTs by electrostatic repulsion. Additionally, a large amount of the CMC surfactant blocks the electronic signal from the CNT surface. In both cases, the sheet and/or electron transfer resistance of the CNT film increase. CMC is superior to the other surfactants, namely, cellulose and sodium cholate, because the optimum mass ratio $[\mathrm{CMC}] /[\mathrm{CNT}]$ is 3 , which is smaller than $[$ cellulose] $/[\mathrm{CNT}]=5$ and [sodium cholate $] /[\mathrm{CNT}]=20 .{ }^{19,20}$ Therefore, dispersion by $\mathrm{CMC}$ is the most efficient method for thin-film formation while maintaining the conductivity and catalytic ability of the CNTs. The CNT-CMC thin film electrode is expected to produce a well-defined and highly reproducible electrochemical signal along with a low background current, and exhibits high stability toward curcumin.<smiles>COc1cc(/C=C/C(=O)CC(=O)/C=C/c2ccc(O)c(OC)c2)ccc1O</smiles>

Fig. 1 Molecular structure of curcumin. 


\section{Experimental}

\section{Reagents}

Curcumin and CMC were purchased from Sigma-Aldrich (St. Louis, MO, USA). Multi-walled CNTs $(10-30 \mathrm{~nm}$ in diameter, $200 \mathrm{~m}$ in length) were produced by Taiyo Nippon Sanso Corporation (Tokyo, Japan). The real samples of curcumin (powder and liquid) were commercial products purchased from retail stores in Japan.

\section{Electrode preparation}

The electrode was formed by depositing evaporated gold onto a polyethylene terephthalate (PET) film $(60 \times 100 \mathrm{~mm})$. The area of the opening for the working electrode was $9 \mathrm{~mm}^{2}$. Next, the PET film was cut into the desired size $(5 \times 30 \mathrm{~mm})$. The CNTs were dispersed in a $0.3 \% \mathrm{CMC}$ aqueous solution to attain a $0.1 \%$ concentration. Aliquots with a total volume of $5 \mu \mathrm{L}$ of the CNT-CMC solution were dropped onto the evaporated gold surface $(\sim 50 \mathrm{~nm})$, which was then dried. Finally, $5-\mu \mathrm{L}$ aliquots of $1.0 \%(\mathrm{w} / \mathrm{v})$ CMC solution were dropped onto the CNT-CMC layer and allowed to dry. The carbon paste was a mixture of $0.2 \mathrm{~g}$ of graphite powder and $0.4 \mathrm{~g}$ of liquid paraffin. The CNT electrode without the surfactant was a mixture of $0.2 \mathrm{~g}$ of carbon paste and $0.05 \mathrm{~g}$ of CNT powder. These pastes were applied on the gold surface.

\section{Measurement}

Atomic force microscopy (AFM) was conducted in the tapping mode in air using a NanoScope V Dimension Icon stage system produced by Bruker AXS, GmbH (Karlsruhe, Germany). The scanning rate was $0.33-1.0 \mathrm{~Hz}$. Electrochemical measurements were performed as described in our previous studies. ${ }^{17,18}$ The HPLC system (Shimadzu LC-20AD Kyoto, Japan) consisted of a quaternary pump, vacuum degasser, automated sample injector, column oven, and system controller. The separation column was a reversed-phase column $(4.6 \mathrm{~mm}$ in diameter and $150 \mathrm{~mm}$ in length, YMC-Pack ODS-A, Kyoto, Japan) with a nominal particle size of $5 \mathrm{~m}$. The mobile phase was a mixture of water, acetonitrile, and methanol. The flow rate, injection volume, and column temperature were $1.0 \mathrm{~mL} \mathrm{~min}^{-1}, 1.0 \mathrm{~L}$, and $40^{\circ} \mathrm{C}$, respectively. The ultraviolet detection wavelength was $420 \mathrm{~nm}$.

\section{Results and Discussion}

Electrochemical study of curcumin at the CNT-CMC electrode

Figure 2 shows AFM images of the electrode surface fabricated by CNT-CMC dispersion in water. The morphology of the electrode showed that the surfactant CMC was adsorbed onto the CNT surface and the dispersed CNTs formed the CNT network. This indicated that the CNTs were well dispersed and that the individual CNTs formed networks with tube-tube junctions. The thinnest fiber-like structure that was observed had an average width of approximately $30 \mathrm{~nm}$, which corresponded to the average diameter of an individual CNT. This observation proved that the CNTs were unbundled and well-dispersed in water.

It is worth noting that $\mathrm{CMC}$ proved to be a suitable surfactant for dispersing the CNTs in aqueous solutions, and thin-film formation via the drop-casting method was simple, rapid, and inexpensive. Figure 3A shows cyclic voltammograms (CVs) of curcumin at the carbon paste, gold, and CNT-CMC electrodes. The CNT-CMC electrode showed a dramatic improvement in

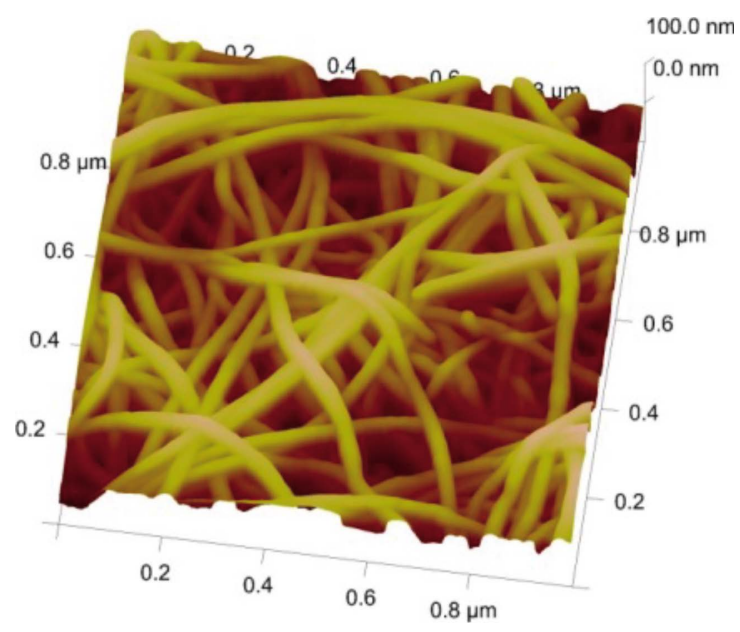

Fig. 2 AFM image of the CNT-CMC electrode.
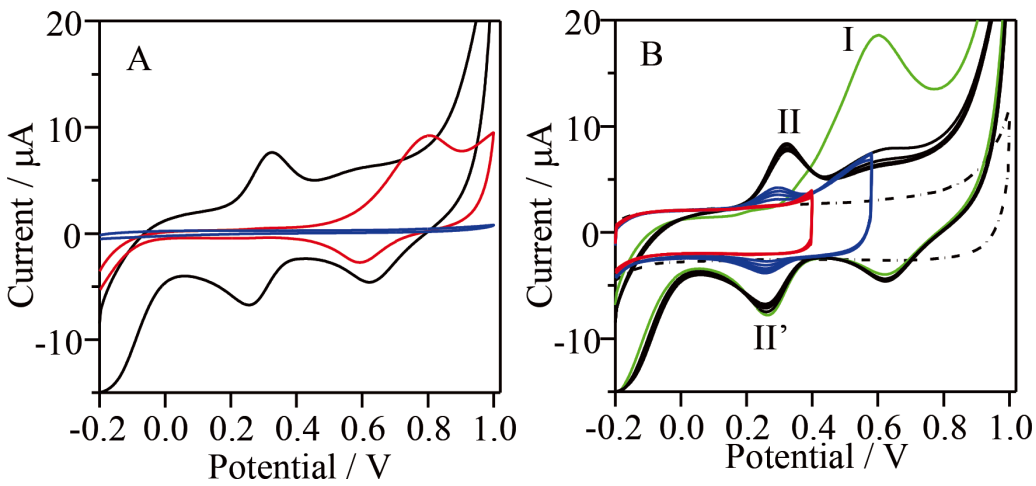

Fig. 3 (A) Cyclic voltammograms (CVs) of curcumin in carbon paste (blue line), gold (red line), and CMC-CNT (black line) electrodes. (B) Consecutive CVs of the CMC-CNT electrode. The broken lines represent the background current. The green line represents the first scan cycle and the black line indicates the second and subsequent cycles. The red and blue lines lie in the scanning range of -0.20 to 0.40 and -0.20 to $0.60 \mathrm{~V}$, respectively. The peak assignments are shown in Fig. 4. (A, B) Concentration is $194 \mu \mathrm{M}$. The electrolyte used was $50 \mathrm{mM} \mathrm{pH} 6.0$ citric acid buffer solution. The scan rate was $0.05 \mathrm{~V} \mathrm{~s}^{-1}$. 
curcumin detection as the oxidation current was considerably larger and the peak position was less positive than that of conventional electrodes. The CNT-CMC dispersion facilitated thin-film formation and also enhanced the electrochemical catalytic ability of the electrode.

The successive $\mathrm{CV}$ profiles and peak assignments of the electrochemical response toward curcumin at the CNT-CMC electrode are shown in Figs. 3B and 4. In Fig. 3B, the green line represents the first scan cycle and the black line represents the second and subsequent cycles. The scan range was from -0.20 to $+1.0 \mathrm{~V}$ (vs. $\mathrm{Ag} / \mathrm{AgCl}$ ). The peak profiles differ significantly between the first and second cycles owing to the reaction pathway of curcumin. The first anodic scan from -0.2 to $+1.0 \mathrm{~V}$ revealed a peak at $+0.60 \mathrm{~V}$ (designated as Peak I). On a subsequent cathodic scan from +1.0 to $-0.2 \mathrm{~V}$, the reduced peak was observed at $+0.25 \mathrm{~V}$ (designated as Peak $\mathrm{II}^{\prime}$ ). In the second anodic scan, a new oxidative peak appeared at $+0.30 \mathrm{~V}$ (Peak II). The peak at $+0.60 \mathrm{~V}$ (Peak I) was observed again, albeit with lower peak heights as compared to that in the first cycle. In the third and subsequent scans, the $\mathrm{CV}$ profiles almost overlapped. This could be explained by an electron transferchemical reaction-electron transfer (ECE) mechanism, as shown in Fig. 4. Peak I was assigned to oxidation of the hydroxyl groups of the benzene ring to the catechol group via a phenoxy radical. This step involves both electrochemical (E, two-electron two-proton process) and chemical (C, hydrolysis of radical) processes (see Fig. 4). The phenoxy radical has two mesomeric forms because of the electronic charge distribution as the highest electron density in the benzene ring of the phenoxy radical is located at the ortho-position. In the following step, the radicals are oxidized to intermediate cation mesomers and this transformation also corresponds to Peak I. Subsequently, the two cation mesomers are transformed to the 3,4-dihydroxybenzene and 3,4-dihydroxy-5-methoxybenzene groups by hydrolysis. This transformation is irreversible and similar to that of the

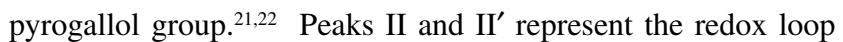
system of the generated catechol group; in other words, the ECE pathway evolves into a reversible electrochemical reaction. This mechanism is depicted in Fig. S1 (Supporting Information) and shows the CVs of caffeic acid and 3,4-dihydroxy-5methoxycinnamic acid as authentic samples. It is evident that the oxidation and reduction potentials of the catechol system fingerprint with Peaks II/II' of curcumin. In this case, Peaks II and II' $^{\prime}$ are assigned to two types of catechol systems. One is the catechol (caffeic acid) route and the other is the methoxycatechol (3,4-dihydroxy-5-methoxycinnamic acid) route (Fig. 4). The oxidation potential difference of the two authentic samples was approximately $0.05 \mathrm{~V}$. In an actual sample (curcumin), these overlapped and, consequently, Peaks II/II' of curcumin were wider than those of the authentic samples. The broad peak could not be attributed to the irreversible system because the peak-to-peak potential separation $\left(\Delta E_{\mathrm{p}}\right)$ of $45 \mathrm{mV}$ was within the range for a quasi-reversible process. Although some researchers have described this mechanism, ${ }^{6,11,23,24}$ this study experimentally validates it for the first time. The curcumin molecule has two $o$-methoxyphenol groups. It is assumed that one benzene ring is oxidized and the other two rings are not oxidised simultaneously because the $\mathrm{CV}$ shape cannot be explained by two-electron reactions. The relationship between $\Delta E_{\mathrm{p}}$ and the electron number $n$ is given by the following equation:

$$
\Delta E_{\mathrm{p}}=59 / n \mathrm{mV}
$$

The value of $n$ was calculated to be less than 4 . The lower

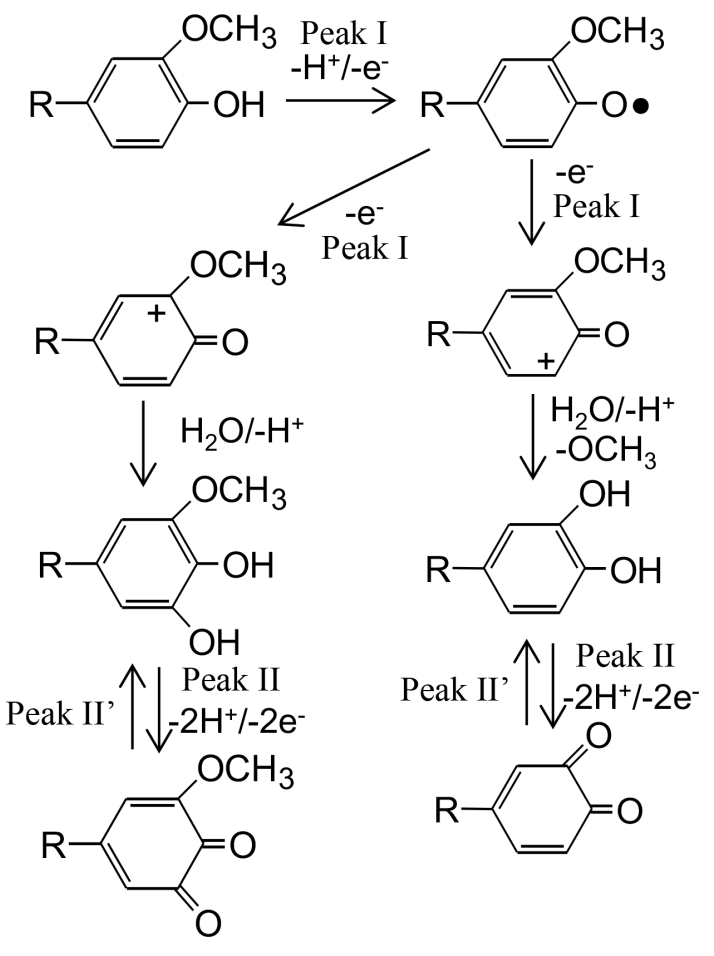

Fig. 4 Electrochemical reaction of curcumin.

sensitivity toward curcumin than that for guaiacol, which has a single $o$-methoxyphenol ring, also supports our assumption (Fig. S2, Supporting Information).

To investigate the ECE mechanism in detail, a new CV experiment was conducted for curcumin. The red lines in Fig. 3B are the successive $\mathrm{CV}$ profiles of curcumin at the CNT-CMC electrode with the potential ranging from -0.20 to $+0.40 \mathrm{~V}$; in other words, the scan direction was reversed at $+0.40 \mathrm{~V}$ before Peak I. There were no distinct peaks in the CV profile and all scans completely overlapped. The blue lines in Fig. 3B are successive CV profiles of curcumin at the CNTCMC electrode with potentials ranging from -0.20 to $+0.60 \mathrm{~V}$; i.e., the scan direction was reversed at the peak position of Peak I. The peak profiles in the first and second cycles were different because of the reaction pathway. Peak II was not observed in the first anodic scan (from -0.2 to $+0.60 \mathrm{~V}$ ). In a subsequent cathodic scan (from +0.60 to $-0.2 \mathrm{~V}$ ), Peak II' was observed. In the second anodic scan, a new Peak II appeared. The current intensities of Peaks II and II' were much smaller than those in the scan from -0.20 to $+1.0 \mathrm{~V}$. This experiment demonstrates that the redox peak pairs II/II' appeared after Peak I. The reduction peak at approximately $+0.65 \mathrm{~V}$ is probably due to the redox reaction of the double bond of the carboxylic acid chain. ${ }^{24}$

The influence of the $\mathrm{pH}$ on the $\mathrm{CV}$ profiles of curcumin was also investigated. The oxidation potential peak (Peaks I and II) positions were negatively shifted upon increasing the $\mathrm{pH}$ from 4.3 to 8.0 . The shifts of all peaks were approximately $60 \mathrm{mV} / \mathrm{pH}$ (Fig. S3, Supporting Information), i.e., the number of transferred protons was equal to the number of transferred electrons, as illustrated in the reaction scheme in Fig. 4. Based on the sensitivity, reproducibility, and stability of the CV profiles, and the fragility of the polyphenols in alkaline media, the following experiment was conducted at $\mathrm{pH} 6.0$.

The effect of the potential scan rate on the CV response for the oxidation of curcumin was investigated within the range of $0.01-0.50 \mathrm{~V} \mathrm{~s}^{-1}$, as shown in Fig. 5A. Both the anodic and 

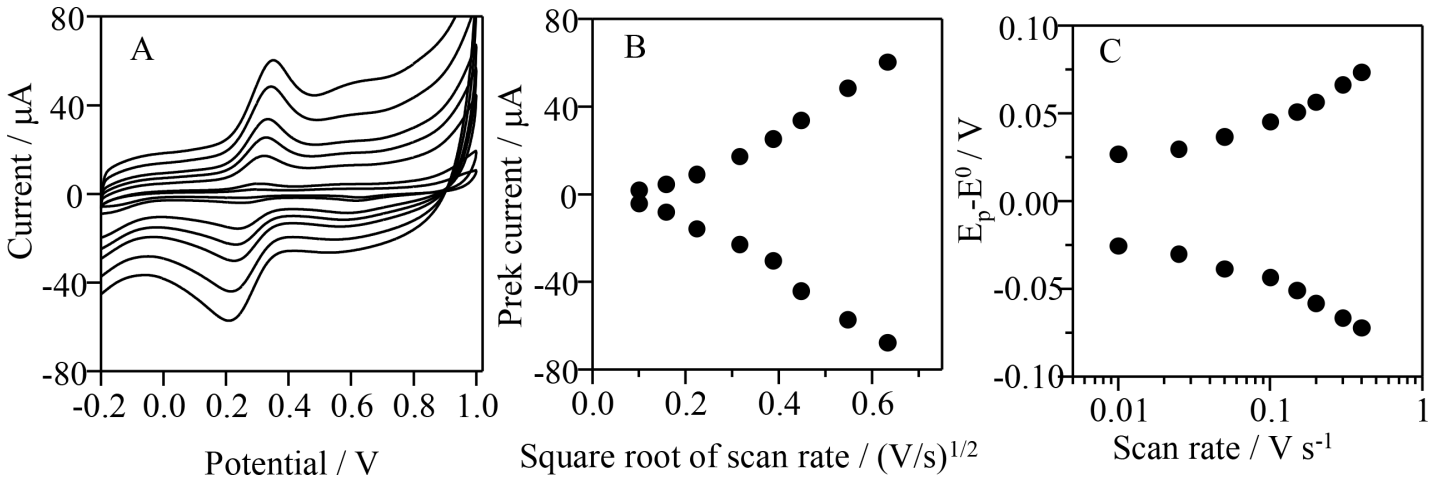

Fig. 5 (A) Cyclic voltammetry of curcumin at the CMC-CNT electrode at various scan rates. Scan rates: $0.01,0.025,0.05,0.10,0.15,0.30$, and $0.40 \mathrm{~V} \mathrm{~s}^{-1}$. (B) Dependence of peak current on the square root of the potential sweep rate. Plots of peak current vs. scan rate. (C) Laviron plot. Concentration is $194 \mu \mathrm{M}$. The electrolyte used was $50 \mathrm{mM}$ pH 6.0 citric acid buffer solution.

cathodic peak currents due to the redox reaction of the catechol group were proportional to the square root of the scan rate within the range of $0.01-0.15 \mathrm{~V} \mathrm{~s}^{-1}$, which was expressed as $I_{\mathrm{pa}}(\mathrm{A})=-10.5+90.6^{1 / 2}\left(\mathrm{~V}^{1 / 2} \mathrm{~s}^{-1 / 2}\right), I_{\mathrm{pc}}(\mathrm{A})=5.4-91.3^{1 / 2}\left(\mathrm{~V}^{1 / 2} \mathrm{~s}^{-1 / 2}\right)$, where $I_{\mathrm{pa}}$ and $I_{\mathrm{pc}}$ are the anodic and cathodic peak currents, respectively. The $I_{\mathrm{p}}$ values were plotted relative to the square root of the scan rate $\left(v^{1 / 2}\right)$ in Fig. $5 \mathrm{~B}$, which indicated that curcumin oxidation was dominated by the diffusion process.

The peak currents of the redox system were proportional to the scan rate at higher scan rates $\left(>0.1 \mathrm{~V} \mathrm{~s}^{-1}\right)$, indicating a limitation owing to the electron-transfer kinetics. In this region, the peak separation increased, indicating that the surface reaction became irreversible with the increasing scan rate. For an irreversible electrode reaction, the relationship between the peak potential and scan rate follows Laviron's law, as given below: ${ }^{25}$

$$
\begin{aligned}
& E_{\mathrm{pa}}=E_{0}^{\prime}-\frac{R T}{(1-\alpha) n F} \ln \left(\frac{R T k_{\mathrm{s}}}{(1-\alpha) n F}\right)+\frac{R T}{(1-\alpha) n F} \ln v \\
& E_{\mathrm{pc}}=E_{0}^{\prime}+\frac{R T}{\alpha n F} \ln \left(\frac{R T k_{\mathrm{s}}}{\alpha n F}\right)-\frac{R T}{\alpha n F} \ln v
\end{aligned}
$$

where, $n, R, T, F, k_{\mathrm{s}}$, and $E_{0}{ }^{\prime}$ are the electron-transfer coefficient, electron number, gas constant, absolute temperature, Faraday constant, standard rate constant of the surface reaction, scan rate, and formal potential, respectively. $E_{0}{ }^{\prime}$ is estimated for the midpoint between $E_{\mathrm{pa}}$ and $E_{\mathrm{pc}}$ at a lower scan rate. At a high scan rate, the plots of $E_{\mathrm{p}} v s$. are linear and given by the following relationship (Fig. 5C):

$$
\begin{aligned}
& E_{\mathrm{pa}}(\mathrm{V})=+0.369+0.0207 \ln v(\mathrm{~V} / \mathrm{s})(r=0.999) \\
& E_{\mathrm{pc}}(\mathrm{V})=+0.186-0.0211 \ln v(\mathrm{~V} / \mathrm{s})(r=0.999)
\end{aligned}
$$

From Eqs. (2) and (4), $(1-\alpha) n$ and $k_{\mathrm{s}}$ were estimated to be 1.2 and $0.60 \mathrm{~s}^{-1}$, respectively. From Eqs. (3) and (5), $n$ and $k_{\mathrm{s}}$ were estimated to be 1.2 and $0.61 \mathrm{~s}^{-1}$, respectively.

\section{Quantification of curcumin in real sample}

The CV profiles of curcumin differed between the first and second cycles. Therefore, we adopted the peak of the third or higher scans for quantitative analysis. Plots of the peak current $v s$. concentrations of curcumin are shown in Fig. 6. Peaks I, II, and $\mathrm{II}^{\prime}$ were quantified as follows. The linear functions for curcumin determination using the peak current $\left(I_{\text {peak }}\right) v s$.

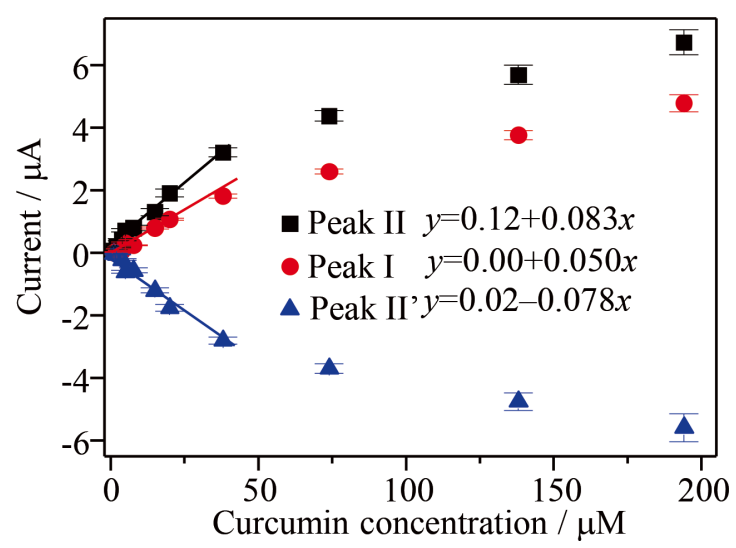

Fig. 6 Plots of oxidation current vs. concentration of curcumin using various peaks in the $\mathrm{CV}$ profiles. The electrolyte used was $50 \mathrm{mM}$ pH 6.0 citric acid buffer solution.

concentration plots were $I_{\text {Peak I }}(\mu \mathrm{A})=0.00+0.050[\mathrm{C}](\mu \mathrm{M})$, $r=0.999$, with a linear range and sensitivity of $1.0-48.0 \mu \mathrm{M}$

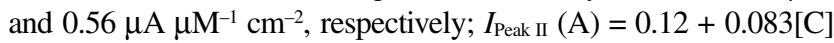
$(\mu \mathrm{M}), \quad r=0.999$, with a linear range and sensitivity of

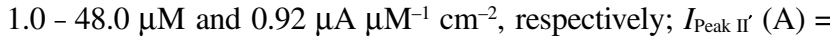
$0.02-0.078[\mathrm{C}](\mu \mathrm{M}), r=0.999$, with a linear range and sensitivity of $1.0-48.0 \mu \mathrm{M}$ and $0.87 \mu \mathrm{A} \mu \mathrm{M}^{-1} \mathrm{~cm}^{-2}$, respectively. The limit of detection (LOD) for curcumin was calculated using the following formula: $\mathrm{LOD}=3 S / M$ (where $S$ is the standard deviation of blank and $M$ is the slope of the calibration plot). The LOD for curcumin detection using the $\mathrm{CV}$ method was $0.084 \mu \mathrm{M}$.

A comparative study of the curcumin-sensing properties of various electrodes is shown in Table 1. It is evident that the performance of the CNT-CMC electrode was similar to that of other reported electrodes. However, the method described in this study is simpler (drop-casting formation), more costeffective (without other functional materials, such as metal nanoparticles), and easier to extend compared to previous methods. The specificity of our procedure is exemplified by the $\mathrm{CV}$ technique, whereas the other methods depend on differential pulse voltammetry or square-wave voltammetry. The advantage of $\mathrm{CV}$ is that (i) a repeat scan keeps the electrode surface clean and, consequently, the reproducibility is enhanced, which is an 
Table 1 Comparison of analytical parameters of various electrodes for curcumin detection

\begin{tabular}{|c|c|c|c|c|c|}
\hline Electrode & Technique & Detection potential/V & Linear range $/ \mu \mathrm{M}$ & Detection limit/ $\mu \mathrm{M}$ & Ref. \\
\hline MWCNT-CMC/Au & $\mathrm{CV}$ & 0.30 & $1.0-48$ & 0.21 & This work \\
\hline Ferrocene NF CP & SWV & 0.28 & $0.1-500$ & 0.024 & 6 \\
\hline Poly-ACBK/GCE & DPV & 0.18 & $0.1-70$ & 0.041 & 7 \\
\hline $\mathrm{LiClO}_{4} \mathrm{GCE}$ & $\mathrm{CV}$ & 0.74 & $9.9-107$ & 4.1 & 8 \\
\hline MWCNT/GC & SWV & 0.7 & $0.01-1$ & 0.005 & 9 \\
\hline $\mathrm{Al}^{3+} / \mathrm{PdNp} / \mathrm{GE}$ & SWV & 0.54 & $0.1-70$ & 0.04 & 10 \\
\hline HMDE & DPV & -1.1 & $0.495-27.6$ & - & 11 \\
\hline Carbon quantum dots & DPV & 0.28 & $0.1-200$ & 0.1 & 12 \\
\hline
\end{tabular}
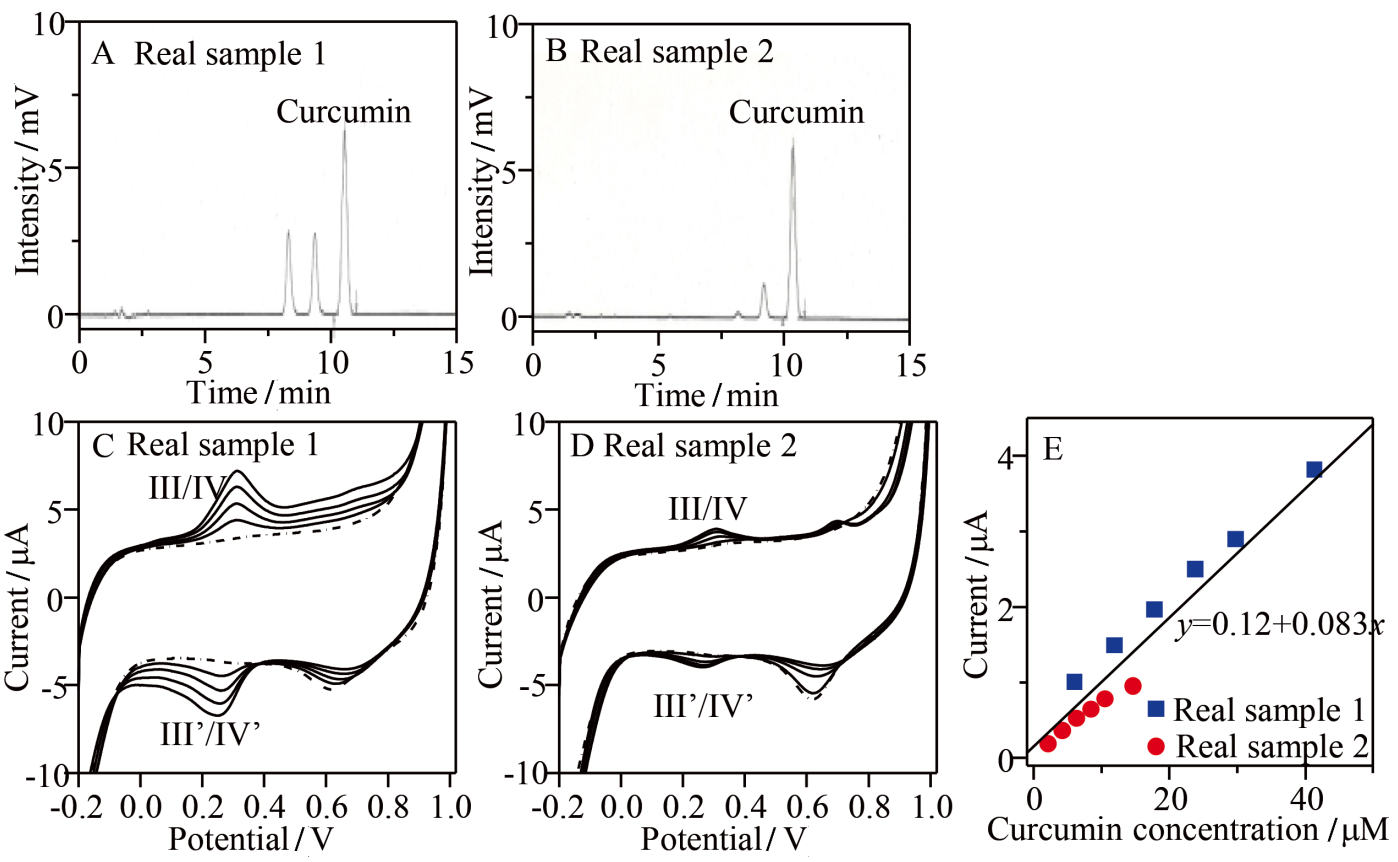

Fig. 7 (A, B) High-performance liquid chromatograms and (C, D) CVs of real samples 1 and 2 for various dilutions. Dilution ratios: (A) 5, (B) 2.5, (C, D) 320, 80, 64, 46. (E) Plot of current $v$ s. diluted concentration based on HPLC data. The black line represents the least-squares fitting line of the solution containing only curcumin in Fig. 6. The electrolyte used was $50 \mathrm{mM} \mathrm{pH} 6.0$ citric acid buffer solution.

advantageous attribute for quantitative analysis, and (ii) it provides both anodic and cathodic currents in a continuous experimental step.

To verify the practicality and reliability of the present procedure, the CNT-CMC electrode was used in the quantification of curcumin in real food samples. Turmeric powders were selected as real samples of curcumin. The powders were dissolved in a $\mathrm{pH} 6.0$ citric acid buffer. The resulting solution was used for both HPLC and electrochemical measurements. The HPLC chromatograms are shown in Figs. 7A and 7B. The concentrations of curcumin in real samples 1 and 2, as determined by HPLC, were 1.9 and $0.67 \mathrm{mM}$, respectively. Figures 7C and 7D show the CVs of the solutions of real samples 1 and 2 at the CMC-CNT electrode at various dilutions. The $\mathrm{CV}$ profiles of the real samples were similar to those of curcumin in Fig. 3. However, Peak I of the real samples was not as clear as that in the analytical standard because it was affected by interfering agents. Figure 7E shows a plot of $I_{\mathrm{p}} v s$. [C] of Peak II based on HPLC data. These plots fitted well with the plotline of standard curcumin in Fig. 6, where the deviation was within 5\%. In other words, there was a strong relationship between the values evaluated by $\mathrm{CV}$ and HPLC. Therefore, we performed electrochemical determinations of curcumin in real food samples. Although this is the first report on the electrochemical determination of curcumin in food samples without the addition of an external standard, we confirmed that the CNT-CMC electrode could be applied to methods involving external standard addition, as shown in Fig. S4 (Supporting Information). The slope of the increment current $v s$. concentration plot corresponds to the standard line in Fig. 7E.

\section{Conclusions}

In this study, the electrochemical quantification of curcumin in real food samples was achieved at the CNT-CMC electrode. This electrode displayed ideal characteristics for curcumin detection, i.e., a high response current and adequate peak separation toward curcumin oxidation. In cyclic voltammograms, 
two oxidation peaks were observed. In the first scan, only the irreversible peak (Peak I) was observed at a higher potential. In the second scan, the reversible redox peak pairs (Peaks II and II') appeared at a lower potential, while the potential of Peak I was decreased. Peak I corresponded to the oxidation of the hydroxyl groups of the benzene ring to the catechol group via a phenoxy radical, while Peaks II and II' were indicative of the redox loop system of the generated catechol group. The electrochemical behavior of curcumin could be explained by the ECE mechanism. The current of Peak II was used to quantify the concentration of curcumin in the linear range of $1-48 \mu \mathrm{M}$ and detection limit of $0.1 \mu \mathrm{M}$. The concentration of curcumin in real food samples, as determined by the CNT-CMC electrode, was consistent with that determined by HPLC.

\section{Acknowledgements}

We would like to thank Editage (www.editage.com) for English language editing.

\section{Supporting Information}

Cyclic voltammograms of caffeic acid, 3,4-dihydroxy-5methoxycinnamic acid, and guaiacol, plot of peak position versus $\mathrm{pH}$ of curcumin, and standard addition experiment. This material is available free of charge on the Web at http://www. jsac.or.jp/analsci/

\section{References}

1. T. Tsuda, Food Funct., 2018, 9, 705.

2. M. A. V. Carmo, C. G. Pressete, M. J. Marques, D. Granato, and L. Azevedo, Food Sci., 2018, 24, 26.

3. R. A. Silva-Buzanello, A. C. Feroo, E. Bona, L. CardozoFilho, P. H. H. Araújo, F. V. Leimann, and O. H. Gonçalves, Food Chem., 2015, 172, 99.

4. Z. Chen, L. Zhu, T. Song, J. Chen, and Z. Guo, Spectrochim. Acta, Part A, 2009, 72, 518.

5. R. S. P. Singh, U. Das, J. R. Dimmock, and J. Alcorn, J. Chromatogr., B, 2010, 878, 2796.
6. M. Afzali, A. Mostafavi, and T. Shamspur, Mater. Sci. Eng., $C$, 2016, 68, 789 .

7. J. Peng, K. Nong, and L. Cen, J. Chin. Chem. Soc., 2012, $59,1415$.

8. G. K. Ziyatdinova, A. M. Nizamova, and H. C. Budnikov, J. Anal. Chem., 2012, 67, 651.

9. P. Daneshagar, P. Norouzi, A. A. Moosavi-Movahedi, M. R. Ganjali, E. Haghshenas, F. Dousty, and M. Farhadi, J. Appl. Electrochem., 2009, 39, 1983.

10. S. Çarr, E. Biçer, and E. Y. Arslan, Croat. Chem. Acta, 2015, $88,105$.

11. Z. Stanić, A. Voulgaropoulos, and S. Girousi, Electroanalysis, 2008, 20, 1263.

12. R. M. Shereema, T. P. Rao, V. B. S. Kumar, T. V. Sruthi, R Vishnu, G. R. D. Prabhu, and S. S. Shankar, Mater. Sci. Eng., $C$, 2018, 93, 21

13. M. M. Dávila, M. S. Flores, and M. P. Elizalde, ECS Trans., 2008, $15,447$.

14. S. Murakami, S. Takahashi, H. Muguruma, N. Osakabe, H. Inoue, and T. Ohsawa, Anal Sci., 2019, 35, 529.

15. R. Chokkareddy, G. G. Redhi, and T. Karthick, Heliyon, 2019, 5, e 01457 .

16. M. Arvand, M. Farahpour, and M. S. Ardaki, Talanta, 2018 , 176, 92.

17. H. Muguruma, S. Murakami, S. Takahashi, N. Osakabe, H. Inoue, and T. Ohsawa, J. Agric. Food Chem., 2019, 67, 943

18. S. Takahashi, H. Muguruma, N. Osakabe, H. Inoue, and T. Ohsawa, Electrochemistry, 2019, 87, 242.

19. H. Muguruma, Y. Inoue, H. Inoue, and T. Ohsawa, J. Phys. Chem. C, 2016, 120, 12284.

20. H. Muguruma, H. Iwasa, H. Hidaka, A. Hiratsuka, and H. Uzawa, ACS Catal., 2017, 7, 725.

21. L. P. Souza, F. Calegari, A. J. G. Zarbin, L. H. MarcolinoJúnior, and M. F. Bergamini, J. Agric. Food Chem., 2011, 59,7620 .

22. S. Gunckel, P. Santander, G. Cordano, J. Ferreira, S. Munoz, L. J. Nunez-Vergara, and J. A. Squella, ChemicoBiol. Interac., 1998, 114, 45.

23. S. K. Trabelsi, N. B. Tahar, B. Trabelsi, and R. J. Abdelhedi, Appl. Electrochem., 2005, 35, 967.

24. M. A. N. Mamaia, V. C. Diculescu, E. S. Gil, and A. M Oliveira-Brett, J. Electroanal. Chem., 2012, 682, 83.

25. E. Laviron, J. Electroanal. Chem., 1979, 100, 263. 